\title{
$n$-clusters for $1<n<7$
}

\section{By Landon Curt Noll and David I. Bell}

\begin{abstract}
An $n$-cluster is a set of $n$ lattice points in $\mathbf{R}^{2}$ with no 3 points collinear, no 4 points concyclic, and where all mutual distances are nonzero integers. The initial discoveries of $n$-clusters for $1<n<7$ are reported. A progress report on small 6-clusters and the search for a 7-cluster is described. A question on the impact of the lattice point restriction is raised, and the definition of $n$-cluster is generalized to $\mathbf{R}^{m}$.
\end{abstract}

We begin with some definitions.

$n$-cluster:

a set of $n$ lattice points $\left\{p_{0}, p_{1}, \ldots, p_{n-1}\right\}$ in $\mathbf{R}^{2}$ with no 3 points collinear, no 4 points concyclic, where all mutual distances between points are nonzero integers

extent of an $n$-cluster:

the radius of the smallest circle, centered at $p_{0}$, which contains all $n$ points of the $n$-cluster

prime $n$-cluster:

an $n$-cluster such that

$$
\operatorname{gcd}\left(d_{0,1}, \ldots, d_{0, n-1}, d_{1,2}, \ldots, d_{1, n-1}, \ldots, d_{n-2, n-1}\right)=1,
$$

where $d_{a, b}$ is the distance between $p_{a}$ and $p_{b}$

equivalent $n$-clusters:

two $n$-clusters are equivalent if and only if they can be made identical under the combined operations of rotation, reflection and translation $n$-pack:

a set of $n$ points $\left\{q_{0}, q_{1}, \ldots, q_{n-1}\right\}$ in $\mathbf{R}^{2}$ with no 3 points collinear, no 4 points concyclic, where all mutual distances between points are nonzero integers

equivalent $n$-packs:

two $n$-packs are equivalent if and only if they can be made identical under the combined operations of rotation, reflection and translation.

In [5, D20, pp. 104-105], Richard K. Guy asks:

(a) Are there six points in the plane, no three in a line, no four on a circle, all of whose mutual distances are rational?

It has been shown that there does not exist in $\mathbf{R}^{2}$ an infinite set of noncollinear points with all mutual distances integral [1], [4]. In [5], Guy presents two opposite conjectures:

Received July 27, 1987; revised January 13, 1988.

1980 Mathematics Subject Classification (1985 Revision). Primary 52A37. 
- There is a number $c$ such that any $n$ points whose mutual distances are rational must contain at least $n-c$ which lie on a circle or line.

- Any polygon can be approximated arbitrarily by a rational polygon [2], [3], [9], [10].

John Leech has found infinitely many sets of 7 points with at most 4 points collinear and 4 points concyclic [5].

We believed that we could more readily answer (a) if we restricted our search to lattice points. By restricting our search to lattice points, we were able to take advantage of simpler algorithms. For example, it is easier to determine integer distance than rational distance. In 1983 we began to investigate $n$-clusters for $n>1$. After many days of NS32016 processor time, we computed the $n$-clusters of smallest extent for $1<n<7$ (see Table 1 ).

TABLE 1

$n$-clusters of smallest extent for $1<n<7$

\begin{tabular}{lrll}
\hline$n$ & extent & $n$-cluster \\
\hline 2 & 1 & $(0,0)(1,0)$ \\
3 & 4 & $(0,0)(3,0)(0,4)$ \\
4 & 6 & $(0,0)(3,4)(3,-4)(6,0)$ \\
5 & 56 & $(0,0)(16,30)(-16,30)(0,33)(56,0)$ \\
6 & 1275 & $(0,0)(546,272)(132,720)(960,720)(546,-1120)(1155,-540)$ \\
\hline
\end{tabular}

By the existence of a 6-cluster, the question raised in (a) is answered in the affirmative (see Figure 1). The smallest 6-cluster was independently discovered about the same time by William Kalsow and Bryan Rosenburg [6].

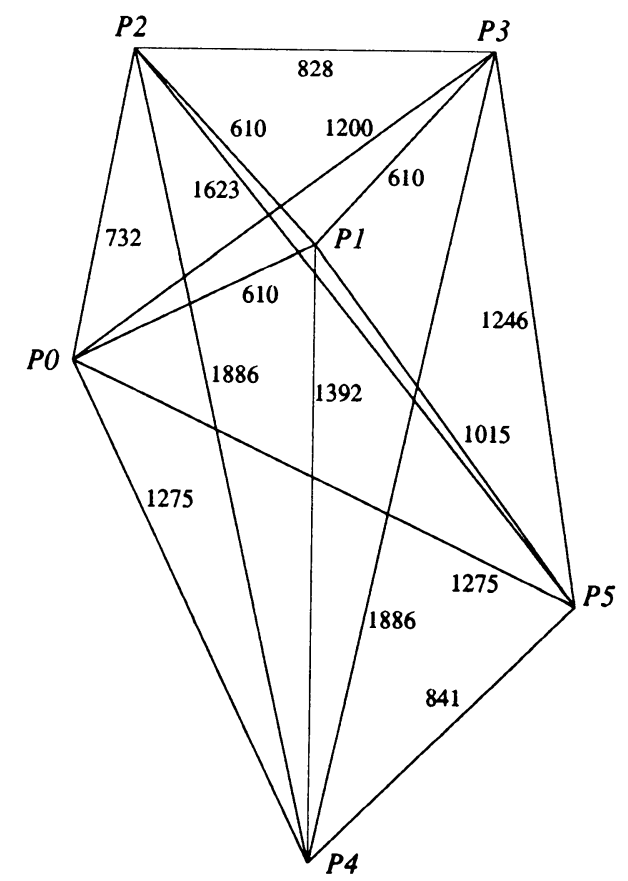

FIGURE 1

The 6-cluster with the smallest extent. 
In 1987, one of use, Landon Curt Noll, ran our program on an Amdahl 5890-300E computer, enabling us to reproduce the results in Table 1 in only 7.10 seconds. We next performed a search for $n$-clusters of extent $<20937$, for $n>5$. The results of this search, given in Table 2, can be summarized as follows:

- There exist 91 nonequivalent prime 6-clusters of extent $<20937$.

- There exists no 7-cluster of extent $<20937$.

- Given any 3 points of a 6 -cluster of extent $<20937$, the triangle formed by these 3 points is a Heron triangle, and is not an Euler triangle [5, D21-22, p. 105].

There is no loss of generality if we restate question (a) as follows:

(b) Are there six points in the plane, no three in a line, no four on a circle, all of whose mutual distances are nonzero integers?

The smallest 6-cluster is not the smallest collection of points that satisfy the question in (b). Recently, Dr. Arnfried Kemnitz showed that the 6-pack shown in Figure 2 is the smallest configuration that satisfies question (b) [7].

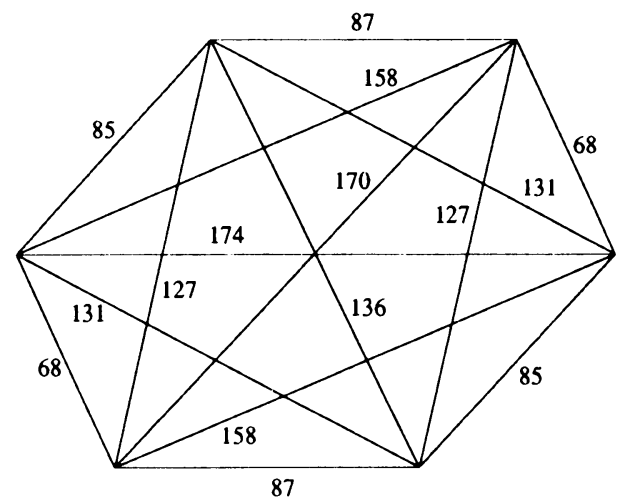

FIGURE 2

The smallest 6-pack.

We wonder how many $n$-packs one ignores when one restricts their search to lattice points. A more precise statement of this question can be found in question (c) below:

(c) Let $P(n, r)$ be the number of nonequivalent $n$-clusters with $p_{0}$ at the origin, and with extent $<r$. Let $Q(n, r)$ be the number of nonequivalent $n$-packs with $q_{0}$ at the origin that lie within a circle of radius $r$ also centered at the origin. What is the value of $P(n, r) / Q(n, r)$, for $n>1$ and $r>0$ ?

It should be realized that our definition of an $n$-cluster in $\mathbf{R}^{2}$ can easily be extended to a more generalized $n$-cluster in $\mathbf{R}^{m}$ called an $n_{m}$-cluster. Such an $n_{m}$-cluster would be defined as follows:

$k_{m}$-flat:

$k$-dimensional affine subspace of $\mathbf{R}^{m}$

$S(k, m, r, p)$ :

$k$-sphere of radius $r$ at origin $p$ in $\mathbf{R}^{m}$

$\left\{x: x, p \in(k+1)_{m}\right.$-flat and $\left.\|x-p\|=r\right\}$ 
$n_{m}$-cluster:

$n$-cluster of order $m$

$\left\{n\right.$ lattice points in $\mathbf{R}^{m}$ :

$m>1 ;$ and

for all $0<k<m$, no $k+2$ points lie in a $k_{m}$-flat; and

for all $0<l<m$, for all $r \in \mathbf{N}$, for all $p$ in $\mathbf{R}^{m}$, no $l+3$ points lie on $S(l, m, r, p)$; and

all mutual distances between points are nonzero integers\}.

For ease of notation, we write $n_{2}$-clusters simply as $n$-clusters.

\section{TABLE 2}

Nonequivalent prime 6-clusters of extent $<20937$.

$(0,0)(399,40)(-1122,-3080)(-600,4480)(3675,-3080)(-8610,-6200)$

$(0,0)(405,252)(-960,720)(570,1760)(1536,1760)(0,3520)$

$(0,0)(546,272)(132,720)(960,720)(546,-1120)(1155,-540)$

$(0,0)(462,440)(-507,-1040)(1431,-1040)(924,-2080)(462,-2520)$

$(0,0)(640,480)(-805,-1764)(-2420,2112)(4070,1656)(-4290,12312)$

$(0,0)(735,392)(-135,1008)(420,-1008)(1155,-616)(1290,-1624)$

$(0,0)(840,189)(252,-1120)(-840,-1575)(1272,-2385)(3780,-2016)$

$(0,0)(805,348)(-1015,348)(-1645,-492)(-105,-1836)(4900,-3552)$

$(0,0)(768,576)(-252,1120)(255,2160)(-1344,2160)(-3840,1120)$

$(0,0)(897,540)(-1023,-1364)(897,-3380)(4257,-860)(8547,-8140)$

$(0,0)(1204,0)(-616,-2112)(429,2340)(-2387,-2940)(1113,-4140)$

$(0,0)(1170,624)(-1525,1548)(-3010,624)(-2240,-2352)(-2240,3600)$

$(0,0)(1088,816)(-1449,0)(2295,-1560)(-855,2640)(2698,4680)$

$(0,0)(1088,816)(1943,-1824)(-1207,2376)(73,-2664)(-1610,-3864)$

$(0,0)(1045,912)(-3050,3096)(-4345,-1608)(-1295,-4704)(-5390,-2520)$

$(0,0)(1045,912)(-3050,3096)(460,5280)(-5390,-2520)(-3960,5280)$

$(0,0)(1008,960)(333,-2040)(-1287,2184)(-2736,2052)(3193,4824)$

$(0,0)(1449,0)(162,-2184)(-2295,-1560)(-1015,3480)(-2698,4680)$

$(0,0)(1449,0)(594,-2640)(-855,-2640)(-3553,2040)(4147,-4680)$

$(0,0)(1330,864)(6055,-2076)(3013,-8316)(8827,564)(-13920,-14616)$

$(0,0)(1560,286)(-672,-1729)(-2520,-1890)(0,-3458)(2940,-6545)$

$(0,0)(1645,492)(-4160,-3120)(-5005,2340)(-4160,7800)(7280,8892)$

$(0,0)(1680,576)(-2280,665)(-2280,-2394)(2280,-2394)(0,3904)$

$(0,0)(1848,161)(624,-2457)(-2376,-1207)(-2376,3168)(-1560,-3744)$

$(0,0)(1848,161)(624,-2457)(-2232,-2015)(-2376,3168)(-1560,-3744)$

$(0,0)(1980,363)(-3060,3213)(-2360,5664)(5780,3213)(1980,14784)$

$(0,0)(2016,840)(2016,-3240)(-5184,2160)(-1309,-7140)(3366,-9240)$

$(0,0)(2016,840)(-720,-4290)(3096,6622)(2016,-7938)(15276,8507)$

$(0,0)(2244,0)(-2145,-5148)(-8970,3952)(1590,11872)(10560,-11088)$

$(0,0)(2244,0)(-2145,-5148)(-8970,-3952)(-8970,3952)(1590,11872)$

$(0,0)(2220,407)(-2652,1161)(-2652,-2464)(-5304,0)(-6480,4032)$

$(0,0)(2223,864)(-3744,-3192)(-1280,-5040)(-6527,3864)(0,7728)$

$(0,0)(2240,1092)(-6076,4557)(-2576,8832)(-9200,0)(-5040,9984)$

$(0,0)(2415,828)(-1155,-2772)(3915,-2772)(-4725,828)(-10275,-6572)$

$(0,0)(2028,1729)(-1404,-4095)(5460,-4095)(2028,-8671)(-13680,-1615)$

$(0,0)(2370,1264)(-2622,2720)(-360,-8096)(7245,-5236)(1305,-11316)$

$(0,0)(2772,0)(1386,-7560)(-9900,-5280)(-6930,-9240)(-15675,-4940)$

$(0,0)(2856,0)(1428,-5104)(1428,-8029)(-2520,-8640)(12768,-12160)$

$(0,0)(2760,805)(2760,-7695)(13680,1615)(18096,-3445)(15960,12325)$ 
TABLE 2 (continued)

$(0,0)(2100,2000)(-2541,4312)(-5775,0)(9009,2288)(-13125,7000)$ $(0,0)(2576,1332)(576,3432)(4160,-4368)(5040,5292)(13455,-5928)$ $(0,0)(2479,1560)(2479,-1560)(11774,0)(12879,-9360)(-5425,-16380)$ $(0,0)(2448,1664)(-3876,2907)(-912,5434)(-5472,-646)(-7980,8379)$ $(0,0)(2184,2080)(-3003,-504)(930,-4760)(-3003,-4004)(-8190,2080)$ $(0,0)(2184,2080)(-3003,-504)(930,-4760)(-3003,-4004)(4290,-7280)$ $(0,0)(2184,2080)(-3003,-504)(-3003,-4004)(4290,-7280)(-8190,2080)$ $(0,0)(3040,684)(0,-3705)(8892,-3705)(8008,11310)(14040,-12441)$ $(0,0)(2604,1953)(0,-11594)(-9672,8246)(5208,-11594)(-4452,19411)$ $(0,0)(2970,1848)(-2065,4956)(-7105,-9312)(3775,11088)(11615,-1512)$ $(0,0)(2970,1848)(0,8976)(2970,-13200)(10075,11160)(-15840,11880)$ $(0,0)(3045,2156)(-2805,4356)(0,8096)(-11970,3040)(-8580,-9504)$ $(0,0)(3965,252)(6055,2076)(3013,8316)(8827,-564)(-13920,14616)$ $(0,0)(3220,2415)(-4620,-441)(1840,-4416)(6600,-6048)(-4620,-12285)$ $(0,0)(3528,1995)(3528,3360)(-4392,5856)(-11232,-1326)(6248,-19680)$ $(0,0)(3360,2520)(-1785,4284)(4998,-4760)(9600,-2800)(-1785,-15120)$ $(0,0)(4120,927)(3060,-4641)(-6320,-4641)(7760,-7809)(4120,-10209)$ $(0,0)(4410,1120)(2040,-4896)(6450,-3776)(-6045,6696)(12495,-10472)$ $(0,0)(3360,3200)(-4620,4851)(-4620,-6160)(-9240,0)(12540,-2299)$ $(0,0)(3780,2835)(-3744,7680)(7056,-6720)(-5684,-11088)(14076,-1455)$ $(0,0)(3840,2880)(-2145,4400)(-3420,15200)(8670,13464)(17280,-7200)$ $(0,0)(3762,3080)(675,-5040)(-5775,3080)(-9537,0)(-6450,8120)$ $(0,0)(4845,540)(-5187,2016)(-2499,-8568)(-9114,8680)(-11424,-8568)$ $(0,0)(5031,492)(5031,11492)(9207,9424)(11895,6344)(-17670,9424)$ $(0,0)(4352,2940)(-3096,-9030)(-8568,5049)(-8568,-10626)(8532,-17751)$ $(0,0)(3927,3740)(4251,5180)(6825,980)(1650,12320)(-15015,-9900)$ $(0,0)(5292,1344)(-3600,5049)(3240,-7776)(-4740,-12032)(10080,-9315)$ $(0,0)(5148,2145)(1196,-6825)(9100,-6825)(14300,-1365)(17020,3735)$ $(0,0)(5148,2145)(-3952,8970)(-9152,3510)(-11872,-1590)(11088,-10560)$ $(0,0)(5304,2210)(0,-7735)(-8976,0)(-5616,8645)(11424,-10880)$ $(0,0)(5304,2210)(-8976,0)(-5616,8645)(2184,-10075)(11424,-10880)$ $(0,0)(5160,2752)(2340,-5859)(-4920,-5166)(-3960,7616)(7800,-3584)$ $(0,0)(5035,3108)(-5040,5292)(8640,7800)(0,12012)(18720,7800)$ $(0,0)(5568,2320)(-11172,0)(10353,13804)(15708,-11200)(3108,19040)$ $(0,0)(4788,3920)(9177,-13680)(14523,-12600)(-2373,-19080)(-19173,1440)$ $(0,0)(6384,113)(0,12325)(13776,11993)(-13776,11993)(6384,17113)$ $(0,0)(6360,1855)(-876,-10640)(2772,12320)(-9240,12320)(-9240,17325)$ $(0,0)(5655,4004)(-6825,-9100)(-1365,-14300)(-15015,-7020)(-10665,13780)$ $(0,0)(6195,3304)(0,-8968)(6195,12272)(-16815,0)(-13965,11704)$ $(0,0)(5250,5000)(-9375,5000)(-12768,1976)(-12768,8024)(7392,-13144)$ $(0,0)(5920,4440)(8120,-6450)(-11396,-2775)(11900,-14535)(20720,-2775)$ $(0,0)(7644,1645)(7956,2635)(-780,-10125)(17556,-2485)(-6636,19355)$ $(0,0)(8712,759)(11000,-8250)(-10168,-11526)(13912,-6534)(-5848,14214)$ $(0,0)(8712,759)(11000,-8250)(-10168,-11526)(12008,-9594)(13912,-6534)$ $(0,0)(8925,1564)(0,-10336)(-12540,10944)(16320,6800)(19635,-1156)$ $(0,0)(9360,1716)(10080,-1848)(15015,4732)(7965,14652)(0,-20748)$ $(0,0)(8800,4290)(-10800,-2430)(-7524,-11985)(0,-16830)(6048,-17640)$ $(0,0)(9471,4428)(3192,12800)(8127,16936)(18207,-4624)(-18753,1096)$ $(0,0)(11781,2508)(-11781,2508)(0,-13200)(15750,0)(-14399,-6468)$ $(0,0)(14756,1365)(-11900,-12495)(9212,-14805)(18500,-5655)(-3100,-19095)$ $(0,0)(13920,5292)(-14784,4070)(0,15732)(-18480,-4158)(18060,9153)$ $(0,0)(12852,8736)(-7480,-15015)(-4256,-17433)(-17776,2457)(0,-18753)$ 
Kevin J. Romero has stated that trivial excitations of cubic crystalline lattices may be considered to form along lines, circles, planes and spheres. He has conjectured that $n_{3}$-clusters might be important in the understanding of nontrivial very long wave length excitations in cubic crystalline lattices, since $n_{3}$-clusters eliminate configurations where such trivial excitations can arise [8].

Acknowledgments. We wish to thank Amdahl Corporation and National Semiconductor Corporation for the use of their computer facilities. We also thank Ronald S. Karr and John Colgrove for their assistance in formatting drafts of this paper and Randall Rathbun for his helpful comments on its contents.

3770 Flora Vista \#1806

Santa Clara, California 95051

1. Norman H. Anning \& PaUl ERdös, "Integral distances," Bull. Amer. Math. Soc., v. 51, 1945 , pp. 598-600.

2. A. S. Besicovitch, "Rational polygons," Mathematika, v. 6, 1959, p. 98.

3. D. E. DAYKIN, "Rational polygons," Mathematika, v. 10, 1963, pp. 125-131.

4. PAUl ERDÖs, "Integral distances," Bull. Amer. Math. Soc., v. 51, 1945, p. 996.

5. Richard K. GUY, Unsolved Problems in Number Theory, Springer-Verlag, New York, 1981.

6. William Kalsow, Private communication.

7. ARNFried KeMnitz, Private communication.

8. KEVIN J. ROMERO, Private communication.

9. T. K. ShenG, "Rational polygons," J. Austral. Math. Soc., v. 6, 1966, pp. 452-459.

10. T. K. SHENG \& D. E. DAYKIN, "On approximating polygons by rational polygons," Math. Mag., v. 38, 1966, pp. 299-300. 